\title{
The Improved LEACH-C Protocol with the Cuckoo Search Algorithm Yun-sheng $\mathrm{GE}^{1,{ }^{*}}$, Jie $\mathrm{KONG}^{1}$ and Kun $\mathrm{TANG}^{1}$ \\ ${ }^{1}$ Guangxi Key Laboratory of Embedded Technology and Intelligent Information Processing, Guilin University of Technology, Guilin, China
}

Keywords: LEACH-C protocol, Cuckoo Search Algorithm, multidimensional space, discrete distribution.

\begin{abstract}
LEACH-C routing protocol chooses the cluster head by simulated annealing algorithm, although the simulated annealing algorithm can optimize network communication distance, however, the convergence of annealing algorithm is very slow. Improved LEACH-C uses the cuckoo search algorithm to optimize network communication distance. To meet discrete distribution of node, the continuous Lévy flight length is discretized. To increase the convergent speed, the annealing probability calculation introduced to the Cuckoo Search Algorithm. The simulation result shows that the improved LEACH-C Algorithm has good convergence speed, reaches low stable objective function values. From the improved LEACH-C algorithm, the cluster heads distribution is relatively uniform, the total WSNs energy consumption is low, and the node death rate gets low.
\end{abstract}

\section{Introduction}

The cuckoo search algorithm (Cuckoo Search CS) is a new intelligent optimization algorithm proposed by University of Cambridge scholars Yang X. S. and Suash D. in 2009[1]. With simple and less parameters, the CS algorithm is concerned by scholars such as Zheng Hongqing[2], who proposed an adaptive step cuckoo search algorithm[3], Song Yujian, who applied the cuckoo algorithm to the multi resources equilibrium optimization[4].

The wireless sensor network(WSNs) consists of a large number of sensors. These sensors usually have limited battery energy. So, to design an energy efficient WSNs routing protocol is a key technology of WSNs[5]. Clustering protocol has been proved to be a valid WSNs routing protocol[6]. $\mathrm{LEACH}-\mathrm{C}$ protocol[7] is a classical centralized WSNs routing protocol. This protocol uses simulated annealing algorithm to calculate the cluster heads, however, the convergence speed of the simulated annealing algorithm is slow and can not guarantee the results to achieve the global optimal.

Compared to simulated annealing algorithm, cuckoo search algorithm uses Lévy flight to search the solution space, and can effectively expand the search scope so as to improve the convergence speed of algorithm. Also the cuckoo algorithm is a swarm optimization algorithm and can reach to the global optimal solution within a shorter period of time.

WSNs nodes are distributed in the discrete multidimensional space, and Lévy flight track is continuous. If the Cuckoo algorithm uses the Lévy flight to find the new cluster head to replace the old one, there exists the problem that when the Lévy flight coordinates for the new point cannot be correspond to the real WSNs node position. In this paper we will adapt the cuckoo algorithm to fit the discrete WSNs nodes 
distribution, and, embed the simulated annealing algorithm into the cuckoo search algorithm to increase the convergence speed and obtain better results.

\section{Related Technologies}

\section{CS Algorithm}

The cuckoo algorithm is based on the following three hypotheses:

(1)Every time a cuckoo lays one egg, and the nest for hatching egg is randomly selected.

(2)In the random search for the nest selection, the best nest will be kept to the next generation.

(3)The probability found by the master is $\mathrm{P} \in[0,1]$, usually is 0.5 . If that happens, the master keeps the nest.

The location update formula for the Cuckoo algorithm is:

$$
x_{i}^{(t+1)}=x_{i}^{t}+\alpha \otimes \operatorname{levy}(\lambda)
$$

Where $x_{i}^{(t)}$ denotes the ith node's position in $\mathrm{t}$ round, $\otimes$ the point-to-point multiplication, $\alpha$ is the step control coefficient, usually takes 1 . Levy $(\lambda)$ is for the Lévy flight search track, the step size obeys the Lévy distribution.

After getting the $x_{i}^{(t+1)}$, we take a random number $\mathrm{r} \in[0,1]$. If $\mathrm{r}>\mathrm{p}$, the $x_{i}^{(t)}$ is replaced by $x_{i}^{(t+1)}$, otherwise keeps $x_{i}^{(t)}$. In the iterative process the best set of nests is always stayed in the next round. The algorithm flow chart is shown in figure 1.

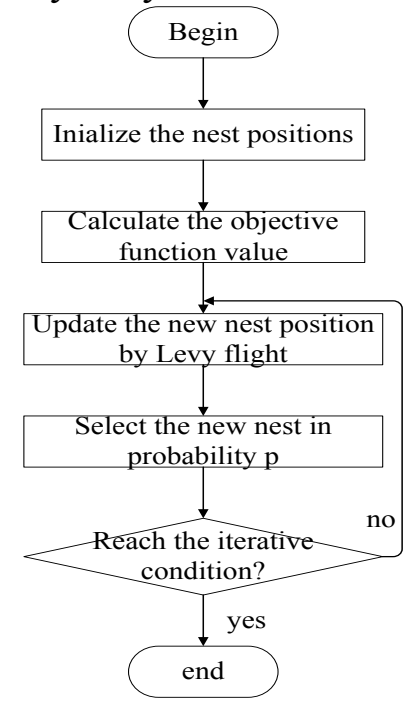

Figure 1 cuckoo algorithm flow chart.

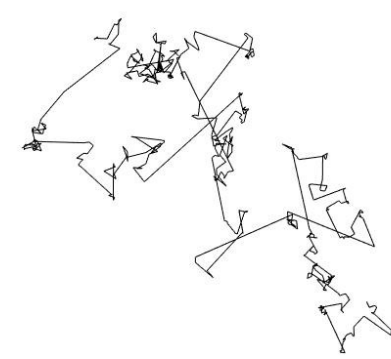

Figure. 2 the Lévy flight trajectory including 500 steps

\section{Lévy Flight Track Distribution with Lévy Flight Length}

In the cuckoo search algorithm, Lévy flight length $s$ obeys Lévy distribution. It can be calculated by the following formula:

$$
s=\frac{u}{|v|^{1 / \beta}}
$$


Where $u$ and $v$ obey standard normal distribution.

$u \sim N\left(0, \partial_{u}^{2}\right), v \sim N\left(0, \partial_{v}^{2}\right)$

For

$\partial_{u}=\left\{\frac{\Gamma(1+\beta) \sin (\pi \beta / 2)}{\Gamma[(1+\beta) / 2] \beta 2^{(\beta-1) / 2}}\right\}^{1 / \beta}, \partial_{v}=1$

Where $\Gamma(\beta)$ is Gamma function.

The Lévy flight step satisfies the heavy-tailed stable distribution. In this random walk, short distance search and the occasional long distance walk, can expand the search range, and it is easier to jump out of the local optimal solution. Figure 2 shows the Lévy flight trajectory in the two-dimensional space.

\section{LEACH-C Protocol}

In LEACH-C protocol, cluster head selection and clustering calculation are fulfilled on the base station. After the base station finishes calculation, it will inform every selected cluster head node in the broadcast form. The energy consumption model of LEACH-C protocol node is as follows:

$$
E_{T}(l . d)=\left\{\begin{array}{l}
l E_{\text {elec }}^{T}+l \varepsilon_{f_{s}} d^{2}, d<d_{o} \\
l E_{\text {elec }}^{T}+l \varepsilon_{\text {mp }} d^{4}, d \geq d_{o}
\end{array}, \quad E_{R}(l)=l E_{\text {elec }}^{R}\right.
$$

Among them, $E_{T}(l, d)$ represents energy consumption that the node sends $l$ bits data to the distance $d$ place, $E_{R}(l)$ for energy consumption to accept $l$ bits data, $E_{\text {elec }}^{T}$ and $E_{\text {elec }}^{R}$ respectively for transmitting circuit and receiving circuit energy consumption for 1 bit data process, $\varepsilon_{f s}$ and $\varepsilon_{m p}$ are transmit amplifier circuit energy consumptions in free space model and multipath attenuation model respectively. When $d<d_{0}$ the free space model is used, otherwise, the multipath attenuation model is adopted, and the $d_{0}$ formula is as follows:

$$
d_{0}=\sqrt{\varepsilon_{f s} / \varepsilon_{m p}}
$$

In each round, the optimal number of cluster heads is calculated with the number of survival nodes in the network, the calculation formula is as follows:

$$
\mathrm{K}_{o p t}=\frac{\sqrt{N}}{\sqrt{2 \pi}} \sqrt{\frac{\varepsilon_{f s}}{\varepsilon_{m p}}} \frac{M}{d_{t o B S}^{2}}
$$

$N$ is the number of nodes distributed in the $M^{*} M$ region, and the $d_{t o B S}$ is the average distance between cluster head to the base station.

From (6), we realize that the energy consumption grows at a geometric rate with the increase of transmit distance. If the total transmit distance is minimized, then the energy consumption is optimal. So, the optimal energy consumption objective function can equivalently transformed to the optimal distance objective function. We can set the optimization objective function as follows:

$$
\min \operatorname{Dist}(\mathrm{S}, \mathrm{J})=\sum_{i=1}^{n} \sum_{j=1}^{k} \operatorname{dist}\left(S_{i}, J_{j}\right)
$$

Among them, $\mathrm{J}$ and $\mathrm{S}$ are non cluster head nodes and cluster heads, and Dist $(\mathrm{S}, \mathrm{J})$ is the distance from the node to the cluster head. 


\section{Improved LEACH-C Protocol with Cuckoo Search Algorithm}

In cuckoo search algorithm Lévy flight step is a continuous scalar. As we want to use the cuckoo algorithm to the WSNs, we first adapt the Lévy flight step to fit the discrete WSNs node distribution. At the same time in cuckoo search algorithm the new nest selection probability remains constant, which possibly causes the iteration oscillation. By the introduction of annealing algorithm, the selection probability of cuckoo search algorithm tends to be small with the increase of iteration round, so as to make the convergence faster.

\section{Lévy Flight Adaption}

At first we suppose that the cuckoo has the ability to detect the nest existence near the Lévy flight track. If the new nest is found, the cuckoo stops to the nest, otherwise continues to fly to the next place.

In every Lévy flight step, if the cuckoo searches all the nests around the new position to detect the nearest nest, it will deteriorate the efficiency of the algorithm. In order to simplify the detection procedure, we divide the WSNs nodes into several blocks. If the Lévy flight track gets into one block, the node in this block is the new nest. The details are described as follows:

First we divide the region into $\mathrm{N}$ blocks, so that the average node number of in each block is 1 . The size of each block is $(\mathrm{M} / \sqrt{N}) *(\mathrm{M} / \sqrt{N})$. Then we design a $\sqrt{N} * \sqrt{N}$ matrix Part, each matrix element contains the number of nodes to the corresponding block and design another $N^{*} N_{m}$ matrix PartNode to store the detail nodes ID, where $\mathrm{N}_{\mathrm{m}}$ indicates the block maximum number of nodes in all blocks. When the cuckoo flies into one block, the corresponding element of Part is checked. If the element value does not equal to 0 , then the PartNode matrix is searched to fix the node ID, else the cuckoo continue to fly to another block. For example, we choose 4 nodes and divide the area into 4 blocks. When the 4 nodes are distributed as shown in Figure 3, the PartNode matrix and the Part matrix store specific contents as shown in table 1.

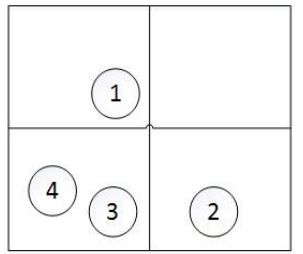

Figure 3 block division for example
Table 1. examples of PartNode matrix and Part matrix storage ble Type Styles

\begin{tabular}{|l|l|l|l|}
\hline \multicolumn{2}{|c|}{ Part } & \multicolumn{2}{c|}{ PartNode } \\
\hline Matrix index & Value & Matrix index & Node ID \\
\hline$(1,1)$ & 1 & 1 & 1 \\
\hline$(1,2)$ & 0 & 2 & NULL \\
\hline$(2,1)$ & 2 & 3 & 3,4 \\
\hline$(2,2)$ & 1 & 4 & 2 \\
\hline
\end{tabular}

\section{Cuckoo Algorithm with Embedded Annealing}

The cuckoo search algorithm chooses the new node with constant probability $p$. This $p$ choosing idea can make the algorithm jump out of the local optimal solution in the initial iteration stage. With the iterating progress, the solution tends to the stable global optimal one. In that case the probability $p$ should be taken a smaller value. The simulated annealing algorithm can solve this problem well. The selection probability is defined as follows:

$$
p=e^{-\Delta / T}=e^{-\left[f\left(x^{t+1}\right)-f\left(x^{t}\right)\right] / T}
$$


and

$$
T=\frac{e^{-t / 50}}{2}
$$

Where, $T$ represents the temperature, $t$ represents algorithm iterative rounds. With the iterative round increases, $\mathrm{T}$ decreases slowly. $f(x)$ is the objective function value calculated by (8).

\section{Cluster Head Nodes Calculation with the Improve LEACH-C}

The cluster head number $K$ can be got by (7). Initially, n groups are constructed, with $K$ nodes in each group, and the objective function values are calculated using (8). The detail iterative process is described in figure 4.

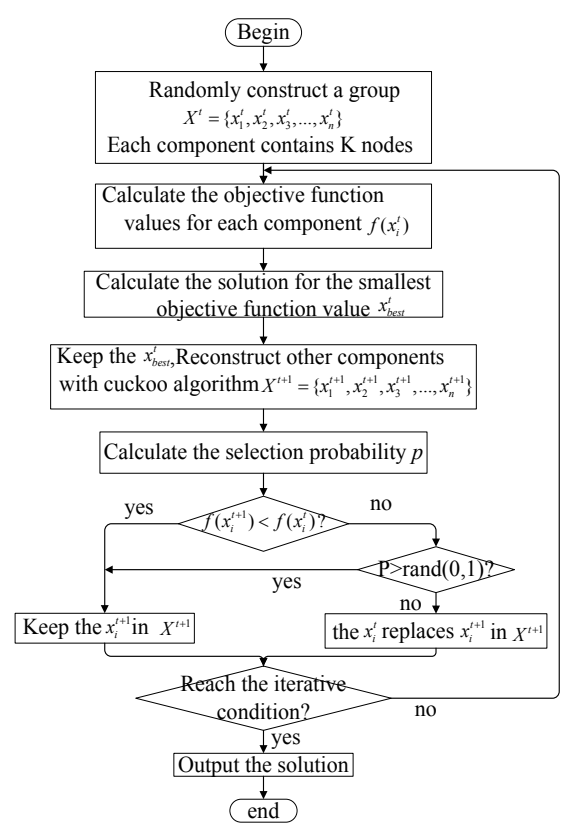

Table 2. experimental parameters

\begin{tabular}{l|l}
\hline \multicolumn{1}{c|}{ parameters } & \multicolumn{1}{c}{ values } \\
\hline Deployment scope & $100 \times 100 \mathrm{~m}$ \\
Base station coordinates & $(100,100)$ \\
Node initial energy & $0.25 \mathrm{~J}$ \\
$\varepsilon_{f s}$ & $10 \mathrm{pJ} / \mathrm{bit} / \mathrm{m}^{2}$ \\
$\varepsilon_{m p}$ & $0.0013 \mathrm{pJ} / \mathrm{bit}^{\mathrm{m}} \mathrm{m}^{4}$ \\
$E_{\text {elec }}^{T}=E_{\text {elec }}^{R}$ & $50 \mathrm{~nJ} / \mathrm{bit}$ \\
Node numbers & 100 \\
$\mathrm{~K}_{\mathrm{opt}}$ & 7 \\
\hline
\end{tabular}

Figure 4 the improved calculation process of cluster head choosing

\section{Simulation and Result Analysis}

In Windows 8 platform, we use the Matlab to carry out simulation experiments. The experimental parameters are shown in table 2. WSNs node distribution and node distribution space block are shown in Figure 5. 


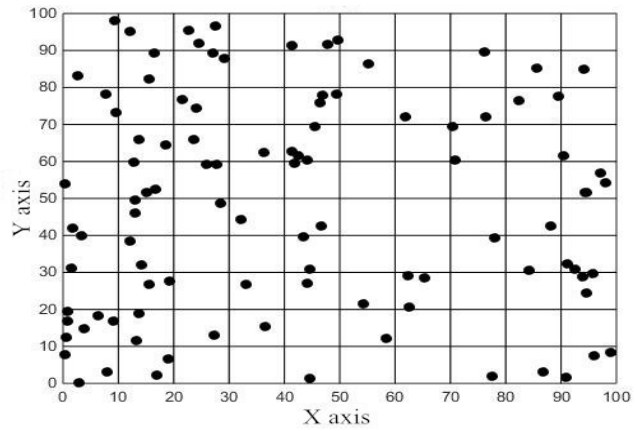

Figure 5 node distribution and block division

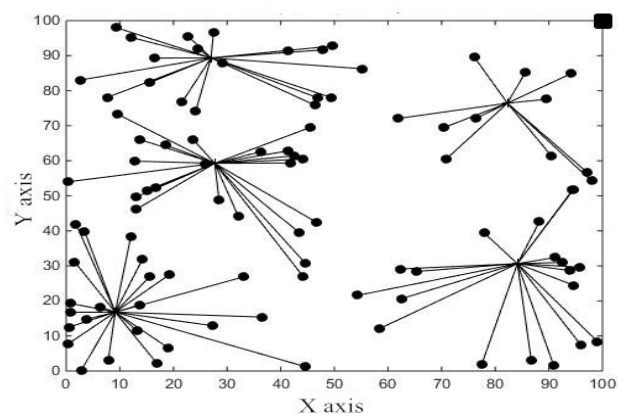

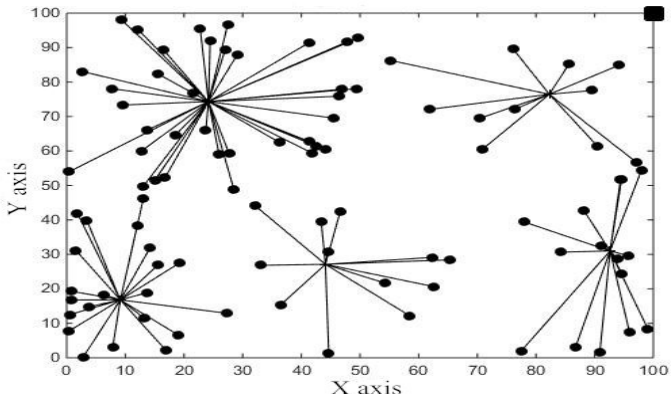

Figure 6 node clustering with original LEACH-C protocol

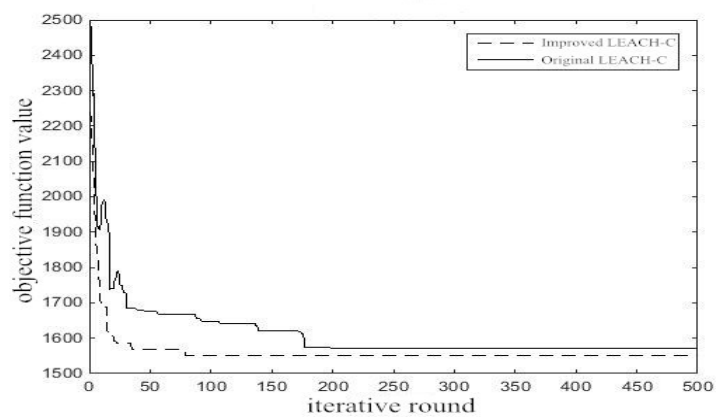

Figure 7 node clustering with improved LEACH-C protocol Figure 8 comparison of the convergence of cluster algorithm

From figure 6 and Figure 7, we can see that the cluster distribution for the improved LEACH-C protocol is relatively uniform. The cluster size on the upper left in figure 6 seems too large, which induces high energy consumption. Figure 8 indicates that the improved LEACH-C protocol has fast converges speed and reaches low energy value.

Figure 9 shows the WSNs taking the improved LEACH-C protocol always keeps higher residual energy at the same round. Because the cluster heads have more uniform distribution for the improved LEACH-C, the energy consumption is less, the lifetime longer, and the total death node number keeps low(figure 10).

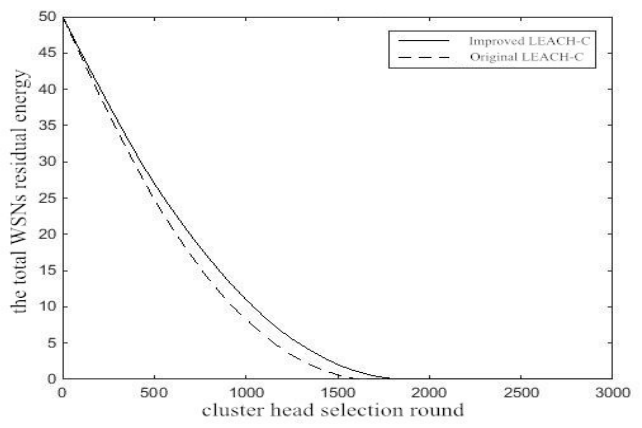

Figure 9 comparison of total WSNs residual energy

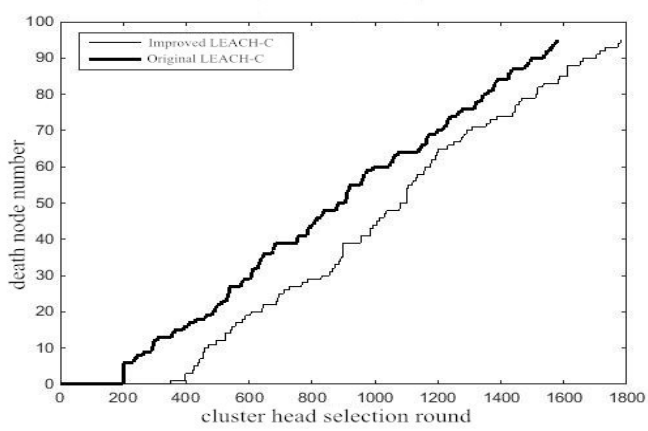

Figure 10 comparison of the number of dead nodes 


\section{Conclusions}

By the introduction of cuckoo search algorithm to the original LEACH-C protocol, first the search area of the cluster head reaches global region, and the cluster head distribution is relatively uniform, which means low energy consumption. At the same time, the use of annealing makes the cuckoo algorithm fast to converge to the stable result. Compared with the original LEACH-C, the low stable energy consumption property improves the total WSNs lifetime, and decrease the node death rate.

\section{References}

[1] Yang XS. Multi objective cuckoo search for design optimization. Computer \& Operations Research, 2013,40(6):1616-1624)

[2] Lan Shaofeng and Liu Sheng, Overview of researh on cuckoo search algorithm, Computer Engineering and Design, Vol. 36, pp. 1063-1067, Apr. 2015.

[3] Zhen Hong-qin and Zhou Yong-quan, Self-adaptive step cuckoo search algorithm, Computer Engineering and Applications, Vol. 10, pp. 68-71, May. 2013.

[4] Song Yujian, Ye Chunming, Huang Zuoxing, Cockoo search algorithm for multi-resource leveling optimization, Journal of Computer Applications, Vol. 34, pp. 189-193, Jan. 2014.

[5] Ankit Thakkar and Ketan Kotecha, Cluster Head Election for Energy and Delay Constraint Applications of Wireless Sensor Network, IEEE Sensors Journal, Vol. 14, pp. 2658-2664, Aug. 2014.

[6] Lin Chushan, Liao Xiaomin, Wang chunyang, Research status and development of routing protocols in Wireless Sensor Networks, China Science and Technology Information, Vol. 20, pp. 134-135, Oct. 2010.

[7] Wendi B. Heinzelman, Anantha P, Chandrakasan, Hari Balakrishnan, An Application-Specific Protocol Architecture for Wireless Microsensor Networks, IEEE transactions on wireless communications, Vol. 1, Oct. 2002 